La situation des familles monoparentales se dégrade. En effet, alors que sur la période 19892000, la part des familles monoparentales au RMI est restée stable, entre $22 \%$ et $23 \%$, à partir de 2001 , elle augmente sensiblement pour atteindre $26 \%$ fin 2002. Les données disponibles (recensement de 1999) ne permettent pas de savoir si ce phénomène est lié à une augmentation généralisée des familles monoparentales ou à une précarisation plus importante de ces familles.

Ce phénomène est d'autant plus préoccupant qu'il cache des réalités différentes. En effet, les familles monoparentales ne représentent pas une catégorie homogène. On observe des différences importantes de précarité selon le sexe, la situation familiale antérieure (mariage ou union libre), le niveau d'études, etc. (10). La femme chef de famille monoparentale, ayant connu une séparation récente, assurant la garde des enfants, dans I'obligation de trouver un logement avec un faible niveau d'études et/ou n'ayant jamais travaillé, ne caractérise qu'une partie de cette population. On peut également distinguer des chefs de familles monoparentales relativement " proches de l'emploi » mais qui, en raison de freins liés à la garde d'enfants et/ou à la mobilité, ne trouvent pas d'emploi ou s'orientent vers des emplois moins qualifiés (femme de ménage à mi-temps).
Références bibliographiques

Algava E., 2002, Les familles monoparentales en 1999, Population, $\mathrm{n}^{\circ}$ 4-5.

Calot G., 1984, Une notion intéressante : I'effectif moyen des générations soumises au risque, Population, $\mathrm{n}^{\circ} 6$.

CNAF (1994-1996), "Statistiques CAF et tous régimes Prestations Familiales », Direction des statistiques, des études et de la recherche/Pôle statistiques.

CNAF (1997-2001), "Statistiques nationales », Direction des statistiques, des études et de la recherche/Pôle statistiques.

CNAF, 2003, "Prestations légales, Aides au logement, Revenu Minimum d'Insertion au 31 décembre 2002 Caisses d'Allocations familiales - Métropole et départements d'Outre-mer ", CNAF, Direction des statistiques, des études et de la recherche/Pôle statistiques.

DIRMI, 1994, Bilan 1993, RMI : Bulletin d'information de la délégation interministérielle au revenu minimum d'insertion, juillet.

Mathieu F., 2001, "Revenu minimum d'insertion au 31 décembre 2000. Caisses d'Allocations familiales Métropole et départements d'Outre-mer ", CNAF, Direction de la recherche, des prévisions et des statistiques/Bureau des statistiques.

Mathieu F., 2002, "Revenu minimum d'insertion au 31 décembre 2001. Caisses d'Allocations familiales Métropole et départements d'Outre-mer ", CNAF, Direction de la recherche, des prévisions et des statistiques, Pôle statistiques.

Mathieu F., 2003, "Revenu minimum d'insertion au 31 décembre 2002. Caisses d'Allocations familiales Métropole et départements d'Outre-mer", CNAF, Direction de la recherche, des prévisions et des statistiques, Pôle statistiques.

\title{
Les mondes vécus des 35 heures
}

\author{
Hervé Defalvard Université de Marne-la-Vallée et Centre d'étude \\ et de l'emploi. \\ Dominique Méda Philosophe, co-auteur de Trente-cinq heures, le temps du \\ bilan, Desclée de Brouver, 2001.
}

Les premières exploitations de l'enquête "Réduction du temps de travail et modes de vie », menée par la Direction de l'animation, de la recherche, des études et des statistiques (DARES) en 2001 auprès $d$ 'un échantillon représentatif de salariés (encadré, p. 80), avaient donné une première idée du jugement de ceux-ci sur les 35 heures: à la question relative aux effets de la réduction du temps de travail (RTT) sur leur vie quotidienne, les salariés ont répondu à $59 \%$ que la RTT l'avait plutôt améliorée, à $13 \%$ qu'elle l'avait plutôt dégradée et à $28 \%$ qu'elle n'avait rien changé pour eux (Estrade, Méda et Orain, 2001). Mais les réponses à la question ouverte qui permettait aux salariés $d^{\prime}$ expliciter leur jugement apportent une image plus précise et plus nuancée des changements induits dans leur vie quotidienne, au travail et hors travail. Elles permettent notamment de mieux comprendre pourquoi c'est sans doute pour leur impact sur la vie familiale que les 35 heures ont été le plus 
L'enquête " réduction du temps

de travail et modes de vie »

L'enquête a été menée en 2000 et 2001 auprès d'un échantillon représentatif de 1618 salariés à temps plein passés à 35 heures avant novembre 1999 (loi Aubry I). La Direction de I'animation de la recherche, des études et des statistiques (ministère de I'Emploi et de la Solidarité) a élaboré le questionnaire, qui concernait les changements intervenus dans le travail et en dehors du travail suite à la mesure de réduction. À la fin du questionnaire, il était demandé aux salariés : "Selon vous, les effets de la RTT sur votre vie quotidienne (au travail et en dehors du travail) ont été plutôt : - Dans le sens d'une amélioration ? - Dans le sens d'une dégradation ? - Cela n'a rien changé ?». Les salariés étaient ensuite invités à expliciter les raisons de leur choix dans une question ouverte.

appréciées. Ces réponses ont, en effet, été soumises à deux types d'exploration, une simple approche par tris croisés et une autre recourant à un logiciel d'analyse de données textuelles, qui se complètent et s'enrichissent.

\section{Amélioration, dégradation ou vie quotidienne inchangée : trois sentiments observés}

\section{Une vie quotidienne qui s'est améliorée pour $59 \%$ des salariés}

Une exploration de chacune des trois postures - amélioration, dégradation, situation inchangée a d'abord été conduite sur la base d'un postcodage en grandes catégories des raisons invoquées par les salariés en réponse à la question ouverte leur demandant d'expliciter leur jugement sur l'impact des 35 heures sur leur vie quotidienne (au travail et en dehors du travail). Pour les $59 \%$ de réponses associant les 35 heures à une amélioration, deux constats émergent. D'une part, ce sentiment est référé exclusivement à la sphère du hors-travail $(1,8 \%$ seulement des salariés considérant que leur vie quotidienne s'est améliorée mentionnent le fait que le travail est plus intéressant). D'autre part, les raisons invoquées pour expliquer ce sentiment font quasiment toutes référence ou bien à l'existence d'un surcroît de temps (plus de temps libre, plus de disponibilité, plus de congés, de plus longs week-ends...) ou bien aux usages de ce surcroît de temps (plus de temps pour la famille, pour le repos, pour les loisirs...). Ainsi, plus de $70 \%$ des salariés indiquant que leur vie quotidienne s'est améliorée se réfèrent, dans leur réponse, à un surcroît de temps et $60 \%$ mentionnent les usages. Les items relatifs à d'autres raisons que celles liées à un surcroît de temps seul ou un surcroît de temps pour un usage précisé sont peu nombreux : quelques salariés parlent de "meilleure qualité de vie », de "travail plus intéressant », mais aussi d'une "diminution du stress».

Il existe peu de différences entre les hommes et les femmes dans la plupart des catégories. Les différences sont plus massives lorsque I'on considère la catégorie socioprofessionnelle (CSP), et opposent souvent les cadres et les non-qualifiés. La prise en considération simultanée de la CSP et du sexe, ainsi que de la présence d'enfants, clive plus fortement les répondants avec des oppositions très fortes entre femmes cadres et femmes non qualifiées, d'une part, et entre les salariés, mais plus encore les femmes ayant des enfants âgés de moins de 12 ans et les autres types de configuration, d'autre part.

\section{$13 \%$ de salariés citent une dégradation de leur vie professionnelle}

En ce qui concerne le sentiment de dégradation (13\% des personnes interrogées ), les constats sont également immédiats : la raison principale expliquant le sentiment de dégradation est l'augmentation de la charge de travail et concerne donc la sphère du travail. L'item le plus fréquemment cité (57 \% des salariés parlant de dégradation) est " moins de temps pour la même charge de travail ». Si on agrège différentes catégories faisant toutes plus ou moins référence à la question des horaires, une proportion identique des salariés considérant que leur situation s'est dégradée (58\%) explique ce sentiment par l'augmentation de la flexibilité des horaires ou la perte de maîtrise de ceux-ci ( "le temps de présence a augmenté », " travaille le samedi, le week-end », " plus grande flexibilité du travail », " augmentation des heures supplémentaires »...). Si, en moyenne, l'intensification du travail est davantage mise en avant comme motif principal de dégradation, proportionnellement, les femmes et les hommes cadres ainsi que les hommes de profession intermédiaire s'en plaignent le plus; les femmes non qualifiées invoquent beaucoup plus que la moyenne les problèmes de flexibilité et d'imprévisibilité plus grande des horaires pour expliciter leur sentiment.

Des motifs divers pour $28 \%$ de salariés déclarant que les $\mathbf{3 5}$ heures n'ont rien changé pour eux

Pour les $28 \%$ de salariés qui déclarent que la mesure n'a rien changé, les motifs sont diversifiés : un groupe $(20 \%)$ indique que la RTT n'a rien changé dans leur «vie quotidienne »- en opposition à des changements plus profonds ou sur d'autres dimensions temporelles - ; un autre 
(12\%) insiste sur le fait que la réduction étant journalière, cela ne change pas grand chose : " II n'y a pas beaucoup plus de temps dégagé » Un troisième groupe $(10 \%)$ indique que " c'est la même chose qu'avant mais avec plus de congés et quelques week-ends ou jours de congés supplémentaires ». Une autre catégorie (14\%) affirme que la charge de travail n'a pas changé ; enfin, une dernière $(13 \%)$ déclare que « le temps dégagé ne correspond pas à celui de la famille, du conjoint » et qu'elle aurait " préféré une autre forme de RTT».

L'approche par les tris croisés permet également de mettre en évidence que les personnes les plus satisfaites de la RTT sont les femmes avec enfants âgés de moins de 12 ans (elles sont $71 \%$ contre une moyenne de $59 \%$ à considérer que leur vie quotidienne s'est améliorée et seulement $4,5 \%$ à considérer que leur vie quotidienne s'est dégradée contre une moyenne de $13 \%$ ). Ces femmes sont également les plus nombreuses à indiquer que, depuis la mise en œuvre de la RTT, la conciliation vie professionnelle et vie familiale s'est améliorée (43\%) ; $63 \%$ d'entre elles déclarent passer davantage de temps avec leurs enfants et $53 \%$ des hommes dans la même situation font de même. Enfin, $41 \%$ des salariés avec enfants âgés de moins de 12 ans déclarent avoir plus de loisirs à I'extérieur avec leur famille.

\section{Des sentiments qui s'affinent en cinq mondes vécus...}

Dans un second temps, on utilise une analyse de données textuelles qui regroupe les réponses en cinq classes de vocabulaire ou mondes lexicaux (1). Ces derniers s'interprètent comme des mondes vécus qui sont alors caractérisés au moyen de neuf variables sociologiques introduites en variables supplémentaires dans I'analyse des classes (encadré, p. 82).

Le monde lexical de la première classe, comprenant $33 \%$ des réponses, associe les 35 heures à plus de temps libre vécu comme un temps partagé avec d'autres dans le cadre familial. L'une des réponses types de cette première classe est : "Ça dégage du temps libre le week-end car on n'a plus besoin de les passer dans les magasins, beaucoup plus de temps pour les enfants». Le salarié idéal typique de ce monde vit dans un foyer avec au moins un enfant âgé de moins de 18 ans, il est plus souvent cadre, profession inter- médiaire ou employé dans le secteur des services, avec un niveau d'enseignement supérieur ou secondaire, et est plus souvent une femme.

Le monde lexical de la deuxième classe, regroupant $19 \%$ des réponses, associe les 35 heures à des congés supplémentaires mais qui sont vécus comme un temps résiduel ou vide, puisque les salariés ne les rapportent à rien de particulier à travers des réponses comme : "Ça m’a donné des jours de congés supplémentaires ». Le salarié idéal typique de ce deuxième monde est plutôt un homme vivant dans un foyer sans enfant avec une conjointe inactive ; il exerce plutôt un travail posté avec une visibilité des horaires qui ne diminue pas. On a reconnu le salarié du monde fordiste dont la norme fut celle de l'emploi masculin à temps plein de la grande industrie.

Le monde lexical de la troisième classe, comprenant $18 \%$ des réponses, associe les 35 heures à un temps dual avec, d'un côté, le temps intensifié du travail et, de l'autre, le temps privé amélioré, où le premier prend le pas sur le second à travers des formulations telles que «Cela a dégradé la qualité de travail, par contre j'ai une amélioration de ma qualité de vie personnelle ». Le salarié de ce troisième monde n'a pas de profil sociologique typé ; on le retrouve de manière uniforme parmi tous les salariés interrogés.

Comptabilisant $17 \%$ des réponses, le monde lexical de la quatrième classe associe les 35 heures à des heures et au temps du faire, selon des perspectives très différentes : "parce que, en fait, le jour RTT je fais mon ménage; donc ça ne change rien " ou " en ce qui concerne les heures, c'est comme si on faisait des heures sup, une petite amélioration financière, une prime en plus ». Là encore, le profil sociologique des salariés de cette classe est peu marqué.

Enfin, le cinquième et dernier monde lexical, ne comptant que $13 \%$ des réponses, associe les 35 heures au temps contraint de la modulation qui s'énonce alors en termes d'horaires : "Pour les horaires en période basse, je fais 2 heures du matin et je finis à 8 heures; en période haute, je fais 22 heures à 6 heures "; "parce qu'on quitte plus tôt le soir en période basse et aussi le matin, c'est moins fatigant, il faudrait que ce soit période basse toute l'année ; surtout, pour une femme, il y a la maison ». Ce temps contraint est ainsi celui des horaires de travail atypiques et irréguliers subis par une petite part des salariés à temps plein (Pélisse, 


\section{La méthode Alceste d'analyse de données textuelles}

En utilisant le logiciel Alceste, on analyse le corpus - formé ici des 1618 réponses des salariés comme une suite de mots pleins (noms, verbes, adjectifs) qui sont les termes ayant un contenu immédiat, en laissant de côté les mots outils qui sont nécessaires à la syntaxe d'une phrase (quand, alors, parce que, etc.). Les coupures opérées dans cette suite de mots pleins relèvent à la fois d'une segmentation naturelle et arbitraire : naturelle car les coupures traduisent le fait que la suite des mots pleins se forme dans les réponses par association d'idées, et arbitraire car elles renvoient à un paramétrage standard selon lequel chaque segment obtenu doit contenir un nombre d'occurrences de mots pleins égal à vingt. Selon I'hypothèse pragmatique, une suite de mots pleins en s'interrompant indique un monde vécu, composé des habitudes du locuteur.

Chaque réponse individuelle est associée à neuf variables qui seront utilisées en variables supplémentaires pour analyser les classes obtenues: I'absence ou la présence d'un conjoint travaillant ou pas, la présence ou non d'enfant(s) âgé(s) de moins de 18 ans, la nature positive, négative ou neutre de la RTT, le sexe, la catégorie socioprofessionnelle, le secteur d'activité, le régime du temps de travail, le niveau d'études et, enfin, la visibilité des horaires. Le tableau des données de base à partir duquel les classes sont calculées suivant la méthode de classification descendante hiérarchique contient ainsi, en colonne, les 262 mots pleins du corpus et, en ligne, les 1622 unités de contexte élémentaire (UCE) du corpus. En raison des réponses relativement courtes des salariés (une phrase ou deux), les UCE du corpus correspondent quasiment ici aux réponses des salariés.

Le calcul des classes procède alors de la manière suivante. Dans une première étape, Alceste calcule la décomposition du corpus en deux classes d'UCE qui, parmi toutes les décompositions possibles, maximise les oppositions, c'est-à-dire le $\mathrm{Khi}^{2}$ des marges. Dans une deuxième étape, Alceste réitère le même calcul en considérant d'abord la classe obtenue comptant le plus d'UCE, afin de chercher la subdivision qui, pour chacune de ces deux classes, maximise les oppositions. Dans I'analyse, la classification descendante hiérarchique aboutit à cinq classes de vocabulaire ou mondes lexicaux. Une classe regroupe des UCE en tant que celles-ci forment des suites de mots pleins qui se répètent dans le corpus et s'opposent à d'autres suites répétées de mots pleins. En raison de la quasi-équivalence entre UCE et réponses des salariés, dans I'article on se réfère directement aux réponses des salariés.
2002). Le salarié idéal typique de ce cinquième monde possède un niveau court d'enseignement, travaille dans les secteurs de l'agriculture ou de l'industrie, vit avec un conjoint qui travaille et, surtout, a vu sa visibilité du temps de travail diminué.

\section{... ouvrant sur une nouvelle lecture des 35 heures}

Les résultats du logiciel Alceste (encadré 2) offrent la possibilité de sortir de la vision classique des 35 heures en termes de gagnants et de perdants. En effet, la démarcation principale qu'ils révèlent oppose deux mondes, celui d'un temps libéré vécu comme un temps partagé en famille et celui d'un supplément de temps résiduel, vide. Alors que, jusque-là, la réduction du temps de travail avait donné lieu à deux grands types de lecture tendant plutôt à la dévaluer, ces résultats ouvrent sur une troisième lecture plus positive des 35 heures.

Une première grille de lecture a largement été développée aussi bien dans les médias que dans les discours politiques, à partir d'une vision purement économique des 35 heures. Trouvant un appui sur le monde fordiste, cette vision va jusqu'à interpréter les 35 heures comme une perte sèche pour le pays, puisqu'elles diminueraient le temps de travail au profit du temps de loisir qui, du point de vue économique ou du PIB, compte pour zéro : il s'agit d'un temps "improductif ». Une seconde lecture a également contribué à forger l'opinion publique sur les 35 heures en mettant en évidence qu'elles ont pu augmenter les inégalités entre travailleurs, puisque les plus satisfaits se trouvent parmi les plus qualifiés et que la proportion de personnes considérant que leur situation s'est dégradée sont en plus grand nombre parmi les peu qualifiés, notamment les femmes, même si les hommes non qualifiés sont $57 \%$ (ce qui est proche de la moyenne de $59 \%$ ) à considérer que leur vie quotidienne s'est améliorée (Estrade, Méda et Orain, 2001). Les mondes vécus du temps de travail intensifié ou du temps de travail contraint ont servi de référence à cette critique sociologique des 35 heures (Pélisse, 2002 ; Lurol et Pélisse, 2001).

La mise en évidence par Alceste d'une population relativement homogène et satisfaite de la RTT en raison des usages familiaux de celle-ci ouvre sur une troisième lecture possible, de nature socioéconomique, en se référant à une association réussie entre le temps de travail et le temps de la famille. Les réponses à d'autres questions de I'enquête RTT et modes de vie vont dans le sens de cette nouvelle perspective. À la question de savoir si elles avaient réfléchi, avant la RTT, à ce qu'elles feraient d'un surcroît de temps libre, $35 \%$ des personnes répondent positivement, les femmes 
avec enfants âgés de moins de 12 ans étant les plus nombreuses (44\%). "À quoi auriez-vous plutôt aimé utiliser ce temps ? » demandait-on à ceux qui y avaient réfléchi : $63 \%$ auraient aimé consacrer ce temps à la famille (89\% pour les femmes avec enfants de moins de 12 ans et $78 \%$ pour les hommes avec enfants de moins de 12 ans). La " famille » devance largement " la maison » (45\%), " soi-même » $(41 \%)$, " les voyages » $(26 \%)$, " les associations » $(12 \%)$.

\section{Un temps producteur de lien, de cohésion et de bien-être}

Les salariés ne souhaitaient donc pas voir leur temps de travail réduit pour disposer de plus de loisirs, mais pour passer davantage de temps avec leur famille. Ce constat est important. En effet, même s'il n'est pas productif de richesse au sens restreint des indicateurs traditionnellement utilisés (au premier chef desquels on trouve évidemment le PIB), ce temps passé avec la famille est indéniablement producteur de lien, de cohésion, de bien-être. Certes, il est délicat de mettre en face des dépenses occasionnées par la RTT des gains de bien-être qui ne peuvent se traduire en termes monétaires. Et cette difficulté permet sans doute de mieux comprendre, d'une part, pourquoi la RTT - qui a répondu à des attentes fortes des personnes pour une meilleure intégration du travail et de la famille - n'a pas été interprétée en ces termes et, d'autre part, comment on pourrait imaginer un usage socialement fort de cette mesure qui ne peut se résumer à une mesure pour l'emploi. Bien qu'elle n'ait pas été présentée pour atteindre cet objectif de meilleure conciliation entre la vie professionnelle et la vie familiale, à travers cette grille de lecture, la RTT apparaît comme une possibilité concrète $d^{\prime}$ 'organiser pour les hommes et les femmes une harmonisation des temps sociaux, notamment ceux du travail et de la famille. Cet objectif est moins anodin qu'il n'y paraît si on sort de la simple opposition entre travail et loisirs et si on prend la mesure de la place que tient aujourd'hui la famille parmi les « valeurs » des Français, laquelle, à travers toutes les enquêtes, vient avant le travail (Méda, 2003). Il I'est également moins si on considère que les taux d'activité féminins pourraient être augmentés si l'organisation du travail et notamment celle des horaires permettaient une meilleure conciliation de la vie professionnelle et de la vie familiale. De ce point de vue, le chantier de l'aménagement du temps de travail est sans doute devant nous.

\section{Bibliographie}

Cette G. et Diev P., 2003, L'impact de la réduction du temps de travail, Futuribles, $\mathrm{n}^{\circ} 285$.

Estrade M.-A., Méda D. et Orain A. (2001), Les effets de la réduction du temps de travail sur les modes de vie, Premières informations et premières synthèses, $\mathrm{n}^{\circ} 21.1$.

Lurol M. et Pélisse M., 2001, 35 heures : les disparités entre hommes et femmes, 4 Pages CEE, $\mathrm{n}^{\circ} 48$.

Méda D., 2003, Manquons-nous de temps?, Interventions économiques.

Méda D. et Orain R., 2002, Transformations du travail et du hors-travail : la formation du jugement des salariés sur la RTT, Travail et Emploi, n 90.

Pélisse J., 2002, À la recherche du temps gagné, les salariés et les 35 heures, Travail et Emploi, n 90.

\title{
La politique de la ville en France et aux États-Unis
}

\author{
\begin{tabular}{l|l} 
Minming Wu & Institut d'études politiques de Paris - Cycle supérieur
\end{tabular} \\ d'aménagement et d'urbanisme.
}

À propos de l'ouvrage de Jacques Donzelot,

Catherine Mével et Anne Wyvekens

Faire société : la politique de la ville aux États-

Unis et en France, Paris, Le Seuil, 2002, 384 pages.

Les villes et la vie américaines apparaissent comme des thèmes bien balisés par les nombreux récits et images véhiculés à leur propos. Ce sentiment d'évidence cache toutefois une compréhension très imparfaite des situations concrètes. L'ouvrage de Jacques Donzelot, Catherine Mével et Anne Wyvekens tente de combler cette lacune en explorant la réalité complexe, trop souvent simplifiée par les discours dominants. Par un travail de 